\title{
Regulation by phosphodiesterase isoforms of protein kinase A-mediated attenuation of myocardial protein kinase D activation
}

\author{
Robert S. Haworth · Friederike Cuello • \\ Metin Avkiran
}

Received: 18 February 2010/Revised: 2 August 2010/Accepted: 12 August 2010/Published online: 20 August 2010

(C) The Author(s) 2010. This article is published with open access at Springerlink.com

\begin{abstract}
Protein kinase D (PKD) targets several proteins in the heart, including cardiac troponin I (cTnI) and class II histone deacetylases, and regulates cardiac contraction and hypertrophy. In adult rat ventricular myocytes (ARVM), PKD activation by endothelin-1 (ET1) occurs via protein kinase $\mathrm{C} \varepsilon$ and is attenuated by cAMP-dependent protein kinase (PKA). Intracellular compartmentalisation of cAMP, arising from localised activity of distinct cyclic nucleotide phosphodiesterase (PDE) isoforms, may result in spatially constrained regulation of the PKA activity that inhibits PKD activation. We have investigated the roles of the predominant cardiac PDE isoforms, PDE2, PDE3 and PDE4, in PKA-mediated inhibition of PKD activation. Pretreatment of ARVM with the non-selective PDE inhibitor isobutylmethylxanthine (IBMX) attenuated subsequent PKD activation by ET1. However, selective inhibition of PDE2 [by erythro-9-(2-hydroxy-3-nonyl) adenine, EHNA], PDE3 (by cilostamide) or PDE4 (by rolipram) individually had no effect on ET1-induced PKD activation. Selective inhibition of individual PDE isoforms also had no effect on the phosphorylation status of the established cardiac PKA substrates phospholamban (PLB; at Ser16) and cTnI (at Ser22/23), which increased markedly with IBMX. Combined administration of cilostamide
\end{abstract}

\footnotetext{
R. S. Haworth $(\square) \cdot$ F. Cuello $~ M$. Avkiran

King's College London British Heart Foundation Centre,

Cardiovascular Division, King's College London,

The Rayne Institute, St Thomas' Hospital,

Lambeth Palace Road, London SE1 7EH, UK

e-mail: robert.haworth@kcl.ac.uk
}

and rolipram, like IBMX alone, attenuated ET1-induced PKD activation and increased PLB and cTnI phosphorylation, while combined administration of EHNA and cilostamide or EHNA and rolipram was ineffective. Thus, cAMP pools controlled by PDE3 and PDE4, but not PDE2, regulate the PKA activity that inhibits ET1-induced PKD activation. Furthermore, PDE3 and PDE4 play redundant roles in this process, such that inhibition of both isoforms is required to achieve PKA-mediated attenuation of PKD activation.

Keywords Cardiac myocyte $\cdot$ PKD $\cdot$ Phosphodiesterase . PKA · Histone deacetylase
Abbreviations
AKAP A-kinase anchoring protein
AR Adrenergic receptor
ARVM Adult rat ventricular myocytes
cTnI Cardiac troponin I
DAG Diacylglycerol
EHNA Erythro-9-(2-hydroxy-3-nonyl) adenine
ET1 Endothelin-1
$\mathrm{G}_{\mathrm{q}} \mathrm{PCR} \quad \mathrm{G}_{\mathrm{q}}$ protein-coupled receptor
HDAC Histone deacetylase
IBMX 3-isobutyl-1-methylxanthine
ISO Isoprenaline
mM199 Modified M199
PDE Phosphodiesterase
PE Phenylephrine
PKA cAMP-dependent protein kinase
PKC Protein kinase $\mathrm{C}$
PKD Protein kinase D
PLB Phospholamban
PLC Phospholipase C 


\section{Introduction}

Protein kinase D (PKD) is a serine/threonine kinase that consists of an $\mathrm{N}$-terminal regulatory domain (containing two cysteine-rich zinc finger-like motifs and a pleckstrin homology domain) and a C-terminal catalytic domain [20, 34]. PKD has been shown to be activated in vitro by diacylglycerol (DAG) and 12-phorbol 13-myristate ester (PMA) [35]. In addition, phosphorylation of Ser744 and Ser748 in the kinase activation loop of PKD by protein kinase $\mathrm{C}$ (PKC) results in DAG-independent catalytic activity [42]. Although PKD can act either in parallel with or downstream of PKC, previous studies suggest that the latter is the principal mechanism of PKD activation in various cell types [7, 39, 42].

Previously, we have shown that PKD regulates myofilament $\mathrm{Ca}^{2+}$ sensitivity, most likely through its phosphorylation of cardiac troponin I (cTnI) $[8,15]$, which we have confirmed recently using mouse myocardium expressing non-phosphorylatable cTnI [5]. In addition, work from the Olson laboratory [14, 37] has shown that PMA and the $\alpha_{1}$-adrenergic receptor (AR) agonist phenylephrine (PE) induce cardiac hypertrophy in neonatal rat ventricular myocytes by a PKD-dependent mechanism, which involves the direct phosphorylation and subsequent nuclear export of histone deacetylase 5 (HDAC5). A role for PKD in HDAC5 redistribution has been supported by studies in failing rabbit and human myocardium [6], and recent evidence from mice with cardiac-specific PKD deletion indicate that PKD is necessary for the full manifestation of pathological cardiac remodelling in response to stress [13]. Taken together, these findings indicate that PKD plays multiple important roles in cardiac myocytes, making it imperative to better understand the mechanisms that regulate its activity in this cell type.

We have previously investigated the roles of $\mathrm{PKC}$ isoforms and cAMP-dependent protein kinase (PKA) in the regulation of $\mathrm{PKD}$ activity in adult rat ventricular myocytes (ARVM) [17]. We found that $\mathrm{PKC} \varepsilon$ plays a predominant role in endothelin-1 (ET1)-induced PKD activation and such activation is inhibited by a PKA-mediated pathway. This counter regulation of PKD by PKA could have important implications, both under normal circumstances where PKA activity is likely to suppress PKD activation and circumstances where PKA activation is impaired, such as in heart failure [22]. Myocardial PKA activity is thought to be controlled in a localised manner through several mechanisms, including the binding of PKA regulatory subunits to A-kinase anchoring proteins (AKAPs) [12] and the maintenance of discrete cAMP pools by the breakdown of cAMP by compartmentalised phosphodiesterase (PDE) isoforms [4, 27, 36, 40]. The principal PDE isoforms that target cAMP in cardiac myocytes are believed to be PDE2,
PDE3 and PDE4 [38], and it has been suggested that these isoforms have distinct roles in regulating PKA activity in different subcellular compartments, and thereby control different downstream events [21, 26-28, 31, 38, 41].

The principal aim of the present study was to determine if the PKA activity that inhibits PKD activation in ARVM is under the control of distinct PDE isoform(s) in a compartmentalised manner. Our findings indicate that in the presence of basal adenylate cyclase activity, PDE3 and PDE4 regulate the PKA activity that inhibits PKD activation in a redundant manner. However, we find no evidence for a specific role for PDE3 and PDE4 in this process, since PKA-mediated inhibition of PKD activation (which is likely to occur at the sarcolemma) and PKA-mediated phosphorylation of cTnI (at the sarcomere) and phospholamban (PLB; at the sarcoplasmic reticulum) were similarly regulated by these PDE isoforms.

\section{Materials and methods}

\section{Materials and animals}

Antibodies for PKD, pSer916 PKD, pSer744/748 PKD and pSer22/3 cTnI were from Cell Signaling Technology, PLB and pSer16 PLB were from Badrilla, and pSer498 HDAC5 was from Abcam. Horseradish peroxidase-conjugated secondary antibodies and enhanced chemiluminescence reagents were from GE Healthcare. Isoprenaline (ISO) and PE were from Sigma Chemical Co. ET1, isobutylmethylxanthine (IBMX), erythro-9-(2-hydroxy-3nonyl) adenine (EHNA), cilostamide, rolipram and H89 were from Merck. M199, penicillin and streptomycin were from Invitrogen. The adenoviral vector used to express full length mouse PKD1 in ARVM was prepared and used as described earlier [8]. Adult male Wistar rats $(250 \mathrm{~g})$ were from B \& $\mathrm{K}$ Universal. The investigation was performed in accordance with the Home Office Guidance on the Operation of the Animals (Scientific Procedures) Act 1986, published by Her Majesty's Stationery Office.

\section{Short term culture and adenoviral infection of ARVM}

ARVM were isolated as described earlier [30, 33], and washed with modified M199 medium [mM199; M199 medium with added penicillin (100 IU/ml), streptomycin (100 IU/ml), L-carnitine $(2 \mathrm{mM})$, creatine $(5 \mathrm{mM})$ and taurine $(5 \mathrm{mM})]$. The cell suspension was centrifuged at $100 \mathrm{~g}$ for $2 \mathrm{~min}$ to pellet the myocytes, which were then resuspended in mM199 medium. To each well of a laminated 6-well culture plate, $2 \mathrm{ml}$ of cell suspension was added and the plates were maintained in a 
humidified $5 \% \mathrm{CO}_{2}$ incubator at $37^{\circ} \mathrm{C}$. After $2 \mathrm{~h}$ of preplating, the medium was aspirated, leaving only adherent cells, and $2 \mathrm{ml}$ of fresh, pre-warmed mM199 medium was added.

Adenoviral infection of cultured myocytes was performed after the initial pre-plating step, in order to elevate PKD expression to a readily detectable level. Myocytes were exposed to adenovirus at a multiplicity of infection of 10 plaque forming units/cell for $2 \mathrm{~h}$ at $37^{\circ} \mathrm{C}$, before the medium containing residual virus was aspirated and replaced with fresh, pre-warmed $\left(37^{\circ} \mathrm{C}\right) \mathrm{mM} 199$ medium. ARVM were maintained in culture for $18 \mathrm{~h}$ before use in experiments.

\section{Pharmacological protocols}

Cells were exposed to ET1 $(100 \mathrm{nM})$, PE $(10 \mu \mathrm{M})$ or vehicle (PBS, $0.1 \%$ volume) for $10 \mathrm{~min}$ prior to harvesting. ISO $(100 \mathrm{nM})$, the non-selective PDE inhibitor IBMX (100 $\mu \mathrm{M}$ in DMSO) or the isoform-selective PDE inhibitors EHNA (PDE2; $10 \mu \mathrm{M}$ in water), cilostamide (PDE3; $10 \mu \mathrm{M}$ in DMSO) or rolipram (PDE4; $10 \mu \mathrm{M}$ in DMSO) were added to cells 10 min prior to ET1 or PE and were present throughout the rest of the protocol. Control cells were exposed to the final concentrations of the appropriate inhibitor vehicle for the equivalent period. In some experiments, cells were first exposed to the PKA inhibitor H89 $(10 \mu \mathrm{M})$ or vehicle (DMSO) for $30 \mathrm{~min}$ prior to subsequent additions. PDE inhibitor concentrations were selected on the basis of previous studies investigating PDE isoform functions in cardiac myocytes [10, 11, 26], while the ISO, PE, ET1 and H89 concentrations were based on our previous work $[9,17]$.

\section{Western blotting}

Cells were washed once with PBS, lysed in Laemmli buffer and protein samples separated by SDS-PAGE. After transfer to nitrocellulose membrane (pSer498 HDAC5 blots) or PVDF membrane (all other blots), western analysis was performed. Where both phosphorylated and total protein were determined, duplicate blots were used. Bound antibody was detected by labelling with horseradish peroxidase-conjugated secondary antibody followed by enhanced chemiluminescence. Phosphorylation status was quantified using a densitometer (BioRad GS-800). To permit comparison between experiments analysed on different gels, all quantitative data were normalised to the vehicle-treated samples exposed to ET1 or PE in each experiment. The corresponding vehicle control value was subtracted from each ET1 or PE value to give the agonistinduced change in signal intensity under each condition. Quantitative data are presented as mean \pm SD from 3-4 independent experiments (as indicated in the legends to each figure).

Measurement of the $\left[\mathrm{Ca}^{2+}\right]_{\mathrm{i}}$ transient and sarcomere shortening

$\left[\mathrm{Ca}^{2+}\right]_{\mathrm{i}}$ transient and sarcomere shortening were monitored simultaneously, essentially as we have described previously [8]. ARVM were cultured overnight on glass coverslips, transferred to a superfusion chamber on the stage of a Nikon Eclipse TE300 inverted microscope and continuously superfused with Tyrode's solution $[\mathrm{NaCl}(137 \mathrm{mM})$, $\mathrm{KCl}(5.4 \mathrm{mM}), \mathrm{CaCl}_{2}(1.0 \mathrm{mM}), \mathrm{MgCl}_{2}(0.5 \mathrm{mM}), \mathrm{N}-2-$ hydroxyethylpiperazine- $N^{\prime}$-2-ethanesulfonic acid $(10 \mathrm{mM}$; $\mathrm{pH}$ 7.4) and glucose $(10 \mathrm{mM})]$. Cells were loaded with the fluorescent $\mathrm{Ca}^{2+}$ indicator fura-2 AM $(2 \mu \mathrm{M}$ for 10-12 min). Sarcomere lengths and $\mathrm{Ca}^{2+}$ transients were measured from single myocytes using IonOptix equipment and software. After a 2 min stabilisation period in Tyrode's solution, PDE inhibitors or vehicle were superfused for $4 \mathrm{~min}$, followed by ISO $(10 \mathrm{nM})$ for $5 \mathrm{~min}$. Quantitative data are presented as the mean \pm SEM for $8-9$ experiments.

\section{In vitro kinase assays}

Following various treatments, cells were lysed and PKD was immunoprecipitated as described earlier [16, 17]. In vitro kinase assays were performed using the immunoprecipitated kinase and recombinant cTnI (100 pmol/reaction) at $30^{\circ} \mathrm{C}$ for $15 \mathrm{~min}$, in the presence of $\left[{ }^{32} \mathrm{P}\right]$-labelled ATP $(100 \mu \mathrm{M})$. The reaction was stopped by the addition of Laemmli sample buffer. cTnI was resolved on a $12 \%$ polyacrylamide gel, which was subsequently dried and subjected to autoradiography.

Statistical analysis

Inter-group comparisons were by analysis of variance (ANOVA), followed by Bonferroni $t$ test. $P<0.05$ was considered significant.

\section{Results}

Non-selective PDE inhibition inhibits ET1-induced PKD activation

We first determined whether non-selective PDE inhibition inhibits ET1-induced PKD activation in ARVM. The phosphorylation status of heterologously expressed PKD at Ser916 (autophosphorylation) and Ser744/748 (activation loop phosphorylation by PKC) was determined by western 
blotting. As illustrated in Fig. 1a and shown quantitatively in Fig. $1 b$, exposure of ARVM to ET1 $(100 \mathrm{nM}$ for $10 \mathrm{~min}$ ) promoted robust phosphorylation of PKD at both the activation loop sites and the autophosphorylation site, reflecting PKC-dependent activation of PKD. Consistent with our previous work [17], pretreatment with the $\beta$-AR agonist ISO (100 nM for $10 \mathrm{~min}$ ) substantially reduced ET1-induced PKD phosphorylation. Pretreatment with the non-selective PDE inhibitor IBMX (100 $\mu \mathrm{M}$ for $10 \mathrm{~min})$ also reduced ET1-induced PKD phosphorylation at both the Ser744/748 and the Ser916 sites to a similar level to that achieved with ISO pretreatment. This suggests that global PDE inhibition in the presence of basal adenylate cyclase activity produces a sufficient increase in PKA activity in the relevant compartment to inhibit PKD activation.

As a further indicator of PKD catalytic activity (in addition to autophosphorylation at Ser916), we used a phosphospecific antibody that recognises phosphorylation of the PKD target site in HDAC5, Ser498. As shown in Fig. 1, this antibody detected ET1-induced increases in the phosphorylation of two proteins, migrating at around $140 \mathrm{kDa}$ (upper band) and $105 \mathrm{kDa}$ (lower band). The ET1-induced increase in phosphorylation of the lower band protein was attenuated by pretreatment with either ISO or IBMX, mirroring the effects of these interventions on PKD
Fig. 1 ET1-induced PKD activation is inhibited by pretreatment with IBMX or isoprenaline. Cultured ARVM were exposed to vehicle, IBMX $(100 \mu \mathrm{M})$ or ISO $(100 \mathrm{nM})$ for $10 \mathrm{~min}$, followed by vehicle (con) or ET1 (100 nM) for

10 min. a Representative western blots of phosphoSer916 PKD, phosphoSer744/748 PKD, phosphoSer498 HDAC5 and total PKD, as indicated.

b Quantitative data for the ET1induced change in phosphoSer916 PKD, phosphoSer744/748 PKD, and the upper and lower bands from the pSer498 HDAC5 blots $(n=4) . * P<0.05$ versus vehicle control

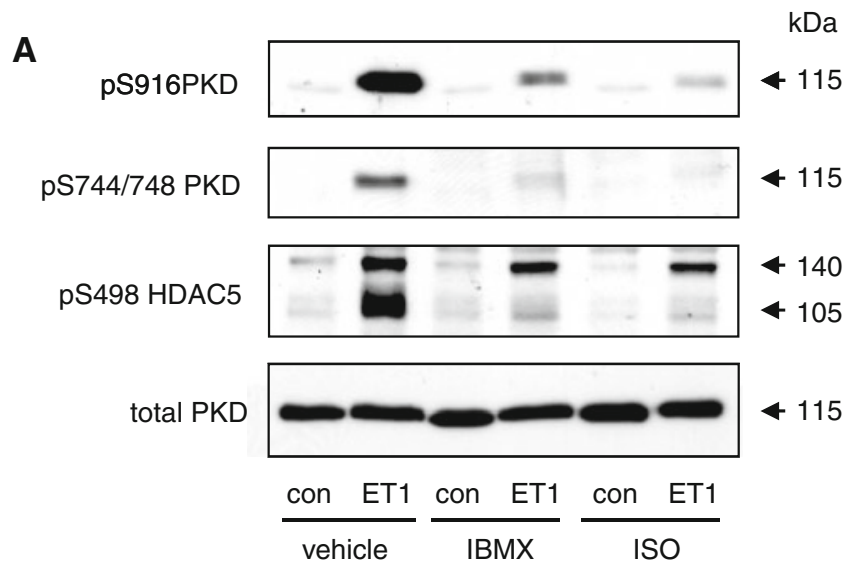

B
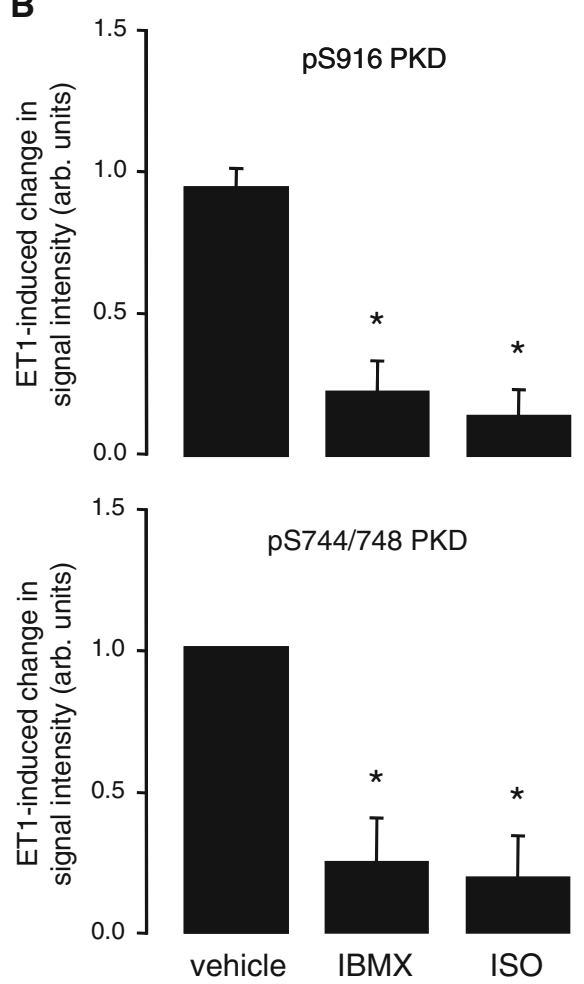

pS498 HDAC5 upper band
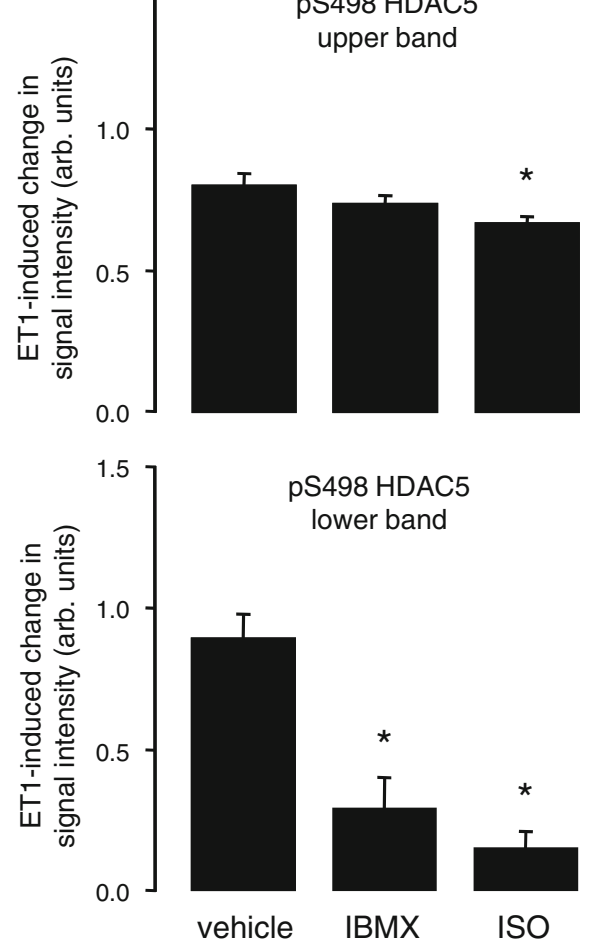
Fig. 2 ET1-induced PKD activation is not inhibited by pretreatment with individual isoform-selective PDE inhibitors. Cultured ARVM were exposed to vehicle, EHNA $(10 \mu \mathrm{M})$, cilostamide $(10 \mu \mathrm{M})$, rolipram $(10 \mu \mathrm{M})$ or ISO (100 $\mathrm{nM}$ ) for $10 \mathrm{~min}$, followed by vehicle (con) or ET1 (100 $\mathrm{nM}$ ) for $10 \mathrm{~min}$. a Representative western blots of phosphoSer916 PKD, phosphoSer744/748 PKD, phosphoSer498 HDAC5 and total PKD, as indicated.

b Quantitative data for the ET1induced change in phosphoSer916 PKD, phosphoSer744/748 PKD, and the lower bands from the pSer498 HDAC5 blots $(n=3)$. $* P<0.05$ versus vehicle control
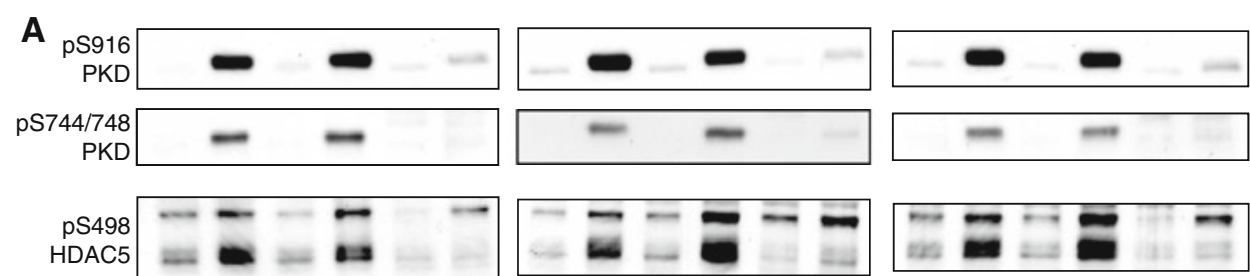
HDAC
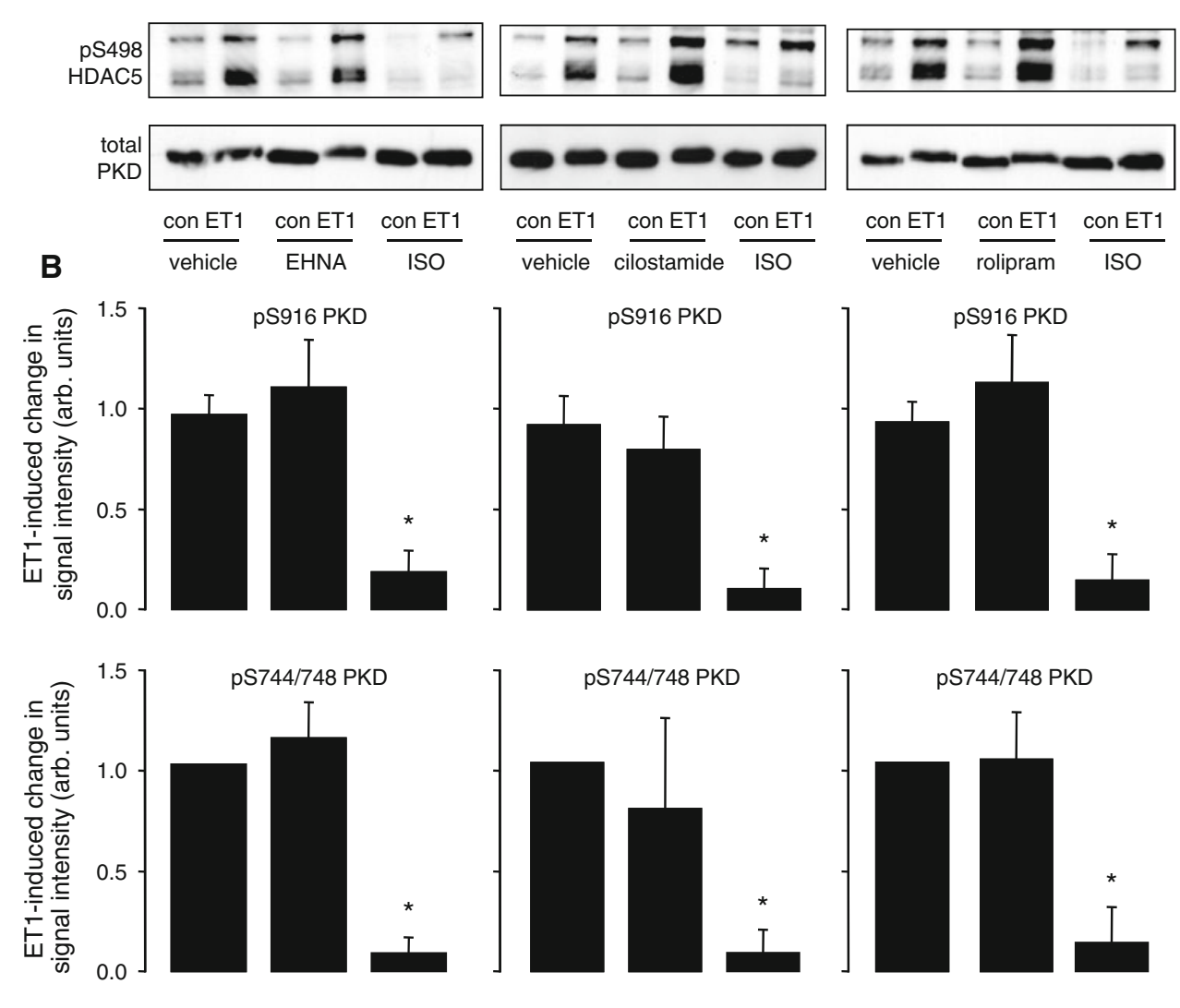

pS744/748 PKD
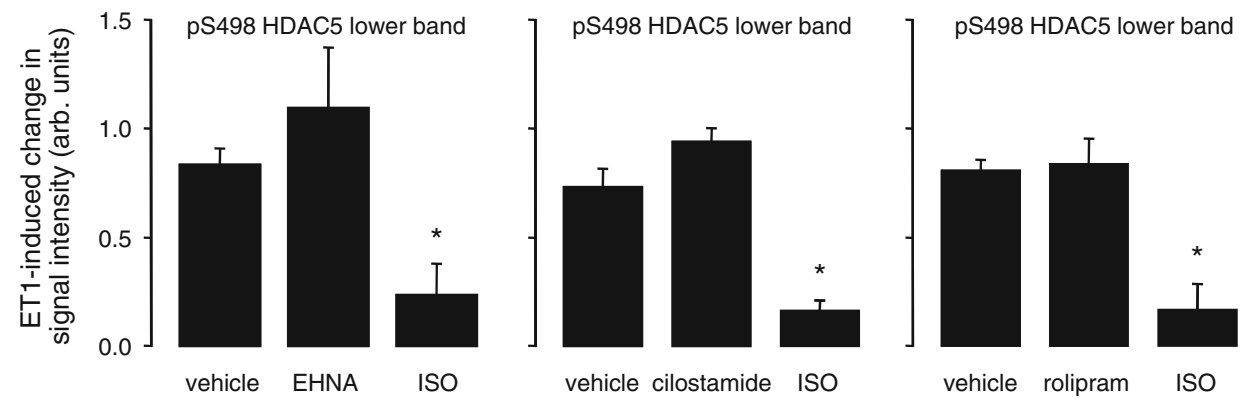

phosphorylation. Interestingly, the ET1-induced increase in phosphorylation of the upper band protein was not significantly affected by pretreatment with IBMX, despite its marked effect on PKD phosphorylation, but was slightly but significantly attenuated by pretreatment with ISO. Although the molecular identities of the proteins representing the upper and lower bands detected by the pSer 498 HDAC5 antibody remain to be established, they are likely to represent different HDAC isoforms (see "Discussion"). Since the phosphorylation status of the lower HDAC band more faithfully reflected the changes in the phosphorylation status of PKD, this was subsequently used as an indicator of PKD catalytic activity.
Selective inhibition of individual PDE isoforms does not inhibit ET1-induced PKD activation

To determine whether any individual PDE isoform is predominantly responsible for regulating the PKA activity that impacts PKD activation, we determined the effects of pretreatment with the PDE2-selective inhibitor EHNA, the PDE3-selective inhibitor cilostamide or the PDE4-selective inhibitor rolipram. As shown in Fig. 2, none of the isoform-selective PDE inhibitors had a marked effect on subsequent ET1-induced PKD phosphorylation and activation, while pretreatment with ISO again produced a consistent inhibitory effect. 
Fig. 3 PKA-mediated phosphorylation of cTnI and PLB is not induced by pretreatment with individual isoform-selective PDE inhibitors. Cultured ARVM were exposed to vehicle, IBMX $(100 \mu \mathrm{M})$, EHNA $(10 \mu \mathrm{M})$, cilostamide $(10 \mu \mathrm{M})$, rolipram $(10 \mu \mathrm{M})$ or ISO $(100 \mathrm{nM})$ for $10 \mathrm{~min}$, followed by vehicle (con) or ET1 (100 nM) for 10 min. Representative western blots of phosphoSer22/23 cTnI, phosphoSer16 PLB and total $\mathrm{PLB}$, as indicated

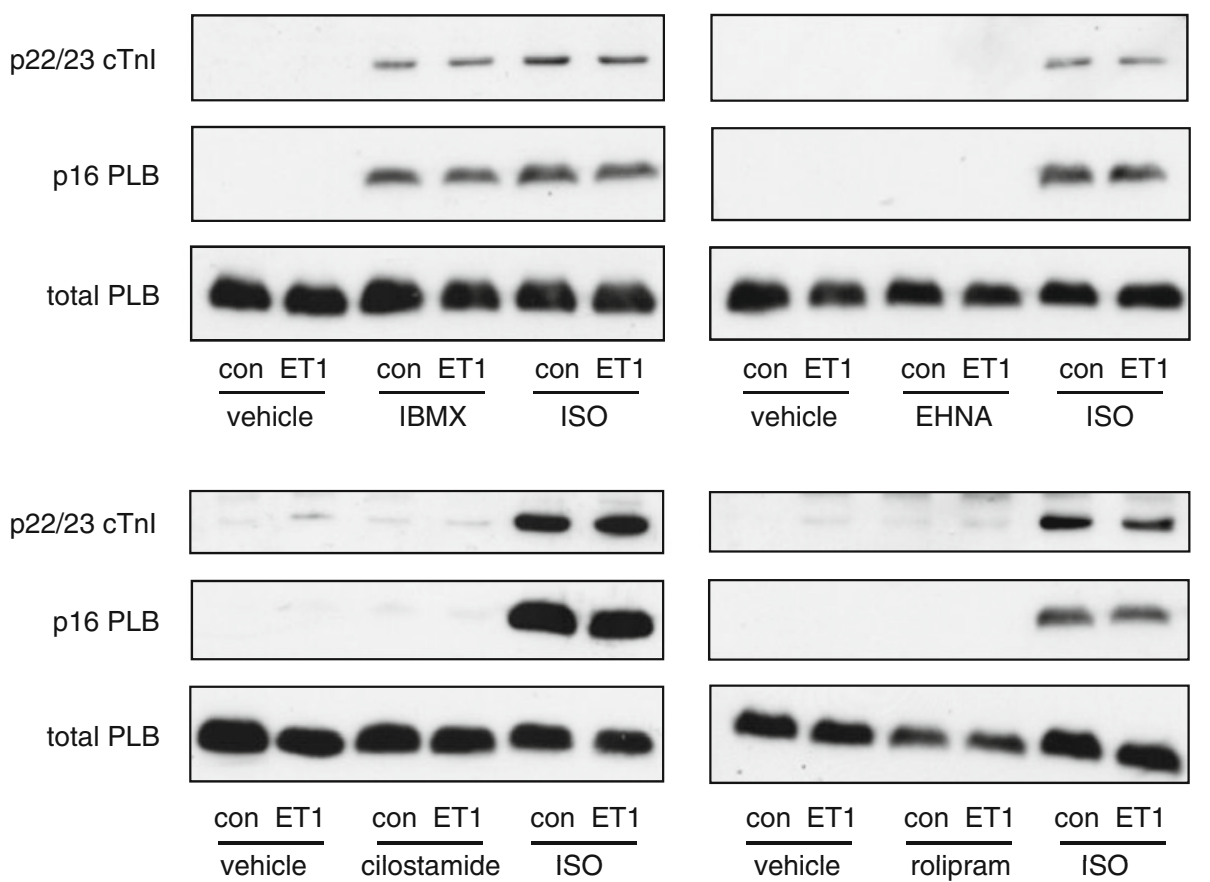

The lack of effect of the isoform-selective PDE inhibitors on ET1-induced PKD activation could be due to their failure to elevate cAMP and activate PKA in the relevant myocyte compartment. To explore whether the isoformselective PDE inhibitors have a different impact on PKA activity in other myocyte compartments, we also examined their effects on the phosphorylation status of cTnI and PLB. As shown in Fig. 3, whilst non-selective PDE inhibition with IBMX or exposure to ISO markedly increased the phosphorylation of cTnI and PLB at their PKA sites, the isoform-selective PDE inhibitors produced little effect. These findings suggest that in the presence of basal adenylate cyclase activity, inhibition of any individual PDE isoform fails to produce a sufficient increase in PKA activity in the myocyte compartments that facilitate the inhibition of PKD activation or the induction of cTnI or PLB phosphorylation.

Combined inhibition of PDE3 and PDE4 is required to induce $\mathrm{cTnI}$ and PLB phosphorylation

We next determined the effects of the simultaneous inhibition of two or more PDE isoforms on PKA activity in the myocyte compartments that facilitate the phosphorylation of cTnI in the sarcomere or PLB at the sarcoplasmic reticulum. As shown in Fig. 4, the PDE2-selective inhibitor EHNA together with either the PDE3-selective inhibitor cilostamide or the PDE4-selective inhibitor rolipram was unable to promote PKA activity in these compartments, as reflected by a lack of effect on the phosphorylation status of cTnI or PLB. However, the combination of the PDE3- and PDE4-selective inhibitors was sufficient to promote phosphorylation of both cTnI and PLB, to an extent similar to that achieved by the combination of all three isoformselective inhibitors or the use of the non-selective PDE inhibitor IBMX. These findings suggest that in the presence of basal adenylate cyclase activity, PDE3 and PDE4 are the principal PDE isoforms that determine PKA activity in the myocyte compartments that facilitate the phosphorylation of cTnI and PLB.

Combined inhibition of PDE3 and PDE4 produces inotropic and lusitropic effects

As the combination of PDE3- and PDE4-selective inhibitors was sufficient to promote phosphorylation of both cTnI and PLB, we investigated the functional effects of this treatment in single cells. The combination of cilostamide and rolipram produced a significant increase in twitch amplitude (Fig. 5a), and this increase was similar in magnitude to that seen with $10 \mathrm{nM}$ ISO (Fig. 5b). The twitch relaxation rate was also increased significantly by exposure to either cilostamide and rolipram or ISO (Fig. 5b). Additionally, the combination of cilostamide and rolipram produced a significant increase in the $\left[\mathrm{Ca}^{2+}\right]_{\mathrm{i}}$ transient amplitude and a significant decrease in the $\left[\mathrm{Ca}^{2+}\right]_{i}$ transient decay time constant (Fig. 5c), with the magnitude of these changes again similar to those seen in response to ISO (Fig. 5d). These findings suggest that, in the presence of basal adenylate cyclase activity, combined inhibition of PDE3 and PDE4 elevates PKA activity in the relevant compartments to a sufficient extent 

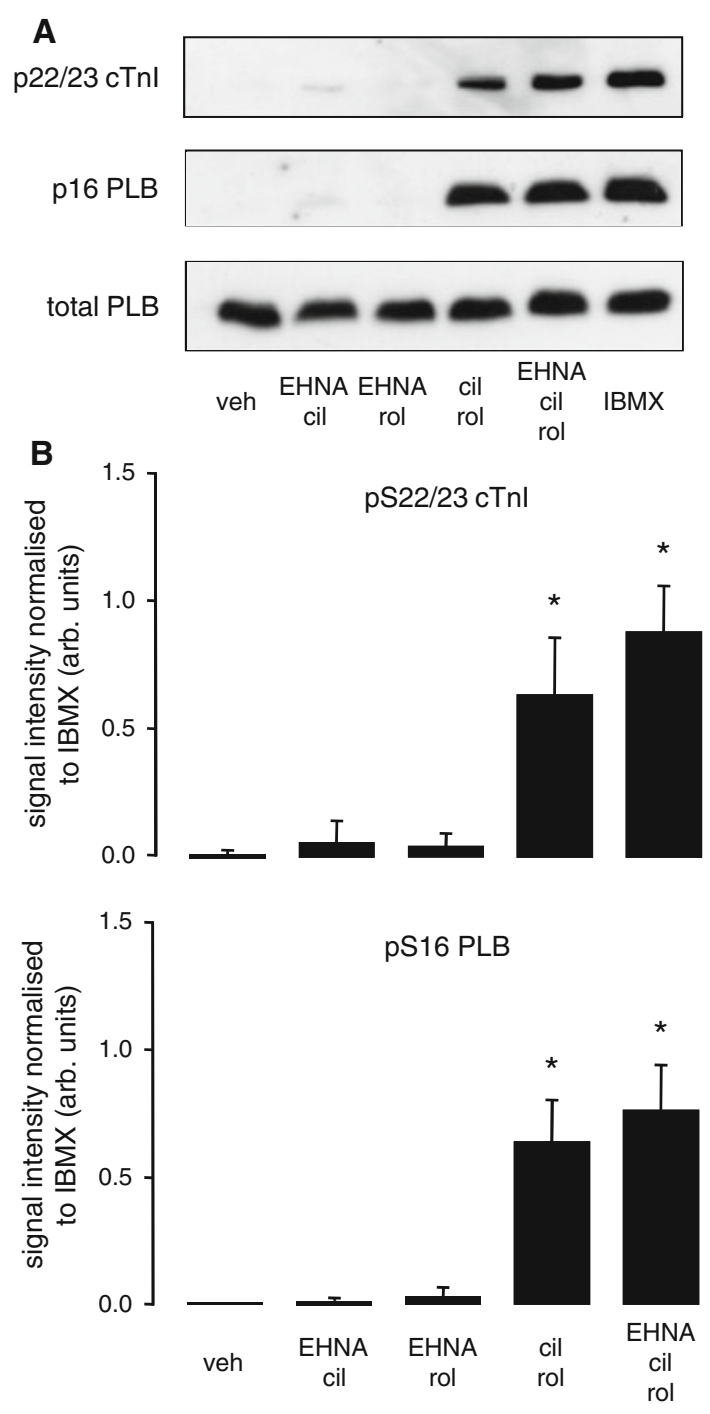

Fig. 4 PKA-mediated phosphorylation of cTnI and PLB requires inhibition of both PDE3 and PDE4. Cultured ARVM were exposed to vehicle, various combinations of EHNA $(10 \mu \mathrm{M})$, cilostamide $(10 \mu \mathrm{M})$ and rolipram $(10 \mu \mathrm{M})$, or $\operatorname{IBMX}(100 \mu \mathrm{M})$ for $10 \mathrm{~min}$. a Representative western blots of phosphoSer22/23 cTnI, phosphoSer16 PLB and total PLB, as indicated $(n=3)$. b Quantitative data for the change in phosphoSer22/23 cTnI and phosphoSer16 PLB normalised to that seen with IBMX $(n=3)$. $* P<0.05$ versus vehicle control

to significantly modify $\mathrm{Ca}^{2+}$ transients and myocyte contraction and relaxation.

Combined inhibition of PDE3 and PDE4 is required to inhibit ET1-induced PKD activation

To determine whether PDE3 and PDE4 also regulate the PKA activity that inhibits PKD activation, we investigated the effects of the isoform-selective PDE inhibitor combinations on ET1-induced PKD activation. As shown in Fig. 6, only pretreatment with the combination of PDE3- selective inhibitor cilostamide and PDE4-selective inhibitor rolipram was able to markedly inhibit ET1-induced PKD activation. These findings indicate that PDE3 and PDE4 are also the principal PDE isoforms that regulate the PKA activity involved in inhibiting ET1-induced PKD activation.

To confirm that the phosphorylation status of Ser916 faithfully reflects the activation status of PKD, we also determined PKD activity by in vitro kinase assays using recombinant cTnI as a substrate. As shown in Fig. 7, exposure of cells to ET1 promoted robust phosphorylation of cTnI by immunoprecipitated PKD in these assays, and this effect was completely blocked by pretreatment of cells with ISO, as we have reported before [17]. Pretreatment with IBMX or the combination of cilostamide and rolipram also substantially reduced cTnI phosphorylation in these assays (Fig. 7), essentially reflecting the changes observed in PKD phosphorylation at Ser916 and Ser744/748 in earlier experiments (Figs. 1,6). These findings confirm that in this preparation, PKD phosphorylation status at Ser916 is a robust index of PKD activity.

PKA mediates the inhibition of ET1-induced PKD activation by combined inhibition of PDE3 and PDE4

To determine whether the combined inhibition of PDE3 and PDE4 inhibits ET1-induced PKD activation through increasing the PKA activity in the relevant compartment rather than through some non-specific effects of cilostamide and rolipram, we investigated the effect of the PKA inhibitor H89 on this response. As shown in Fig. 8, pretreatment with $\mathrm{H} 89$ efficiently blocked the inhibitory effects of combined cilostamide and rolipram, or indeed ISO (as we have shown before [17]), on ET1-induced PKD activation. These findings indicate that combined inhibition of PDE3 and PDE4 inhibits ET1-induced PKD activation through increased PKA activity.

\section{PKA activation inhibits PE-induced PKD activation}

Finally, we determined whether PKA activation by pretreatment with the non-selective PDE inhibitor IBMX or the $\beta$-AR agonist ISO also inhibits PKD activation by members of the $G_{q}$ protein-coupled receptor $\left(G_{q} P C R\right)$ family other than ET1 receptors. As shown in Fig. 9, exposure of ARVM to the $\alpha_{1}$-AR agonist PE induced robust PKD activation. PE-induced PKD activation was inhibited by pretreatment with IBMX or ISO (Fig. 9), mirroring our earlier findings with ET1-induced PKD activation (Fig. 1). These findings suggest that in cardiac myocytes PKA activity may inhibit subsequent PKD activation by multiple $\mathrm{G}_{\mathrm{q}} \mathrm{PCR}$ pathways, including those stimulated by $\alpha_{1}$-ARs and ET1 receptors. 
Fig. 5 Combined inhibition of PDE3 and PDE4 induces inotropic and lusitropic changes. Cultured ARVM were exposed to Tyrode's solution for $2 \mathrm{~min}$, vehicle or combined cilostamide $(10 \mu \mathrm{M})$ and rolipram $(10 \mu \mathrm{M})$ for $4 \mathrm{~min}$, and then ISO $(10 \mathrm{nM})$ for $5 \mathrm{~min}$.

a Representative signalaveraged (14 twitches) contractility recordings for each treatment. b Quantitative data for sarcomere shortening and relaxation rate, as indicated ( $n=8-9$ cells/group).

c Representative signalaveraged (14 twitches) $\left[\mathrm{Ca}^{2+}\right]_{\mathrm{i}}$ transient recordings for each treatment. d Quantitative data for changes in $\left[\mathrm{Ca}^{2+}\right]_{i}$ transient amplitude and decay time constant, as indicated $(n=8-9$ cells/group). $* P<0.05$ versus vehicle control
A

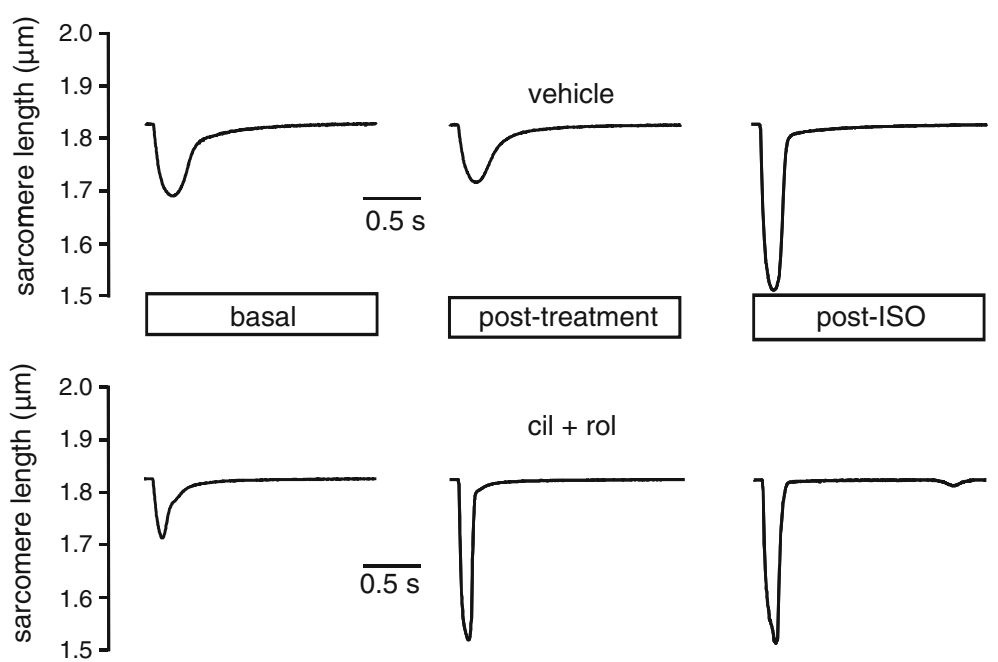

B

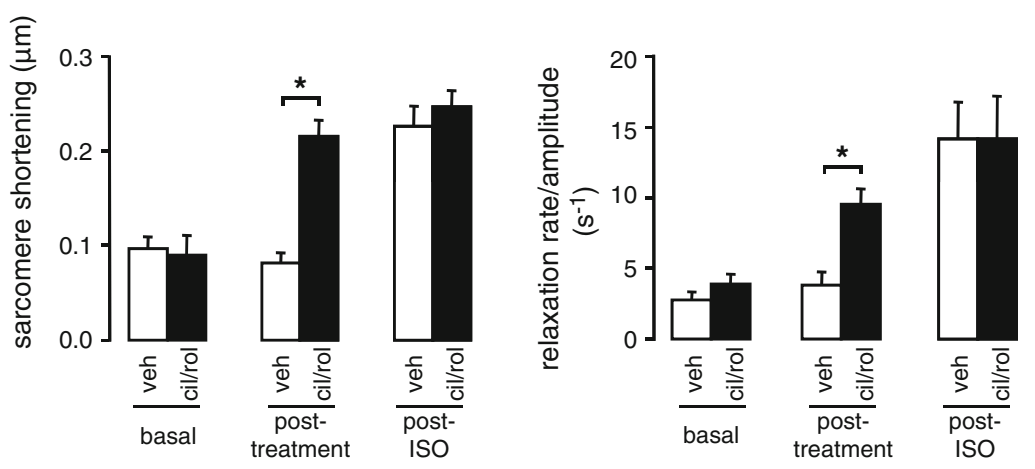

C

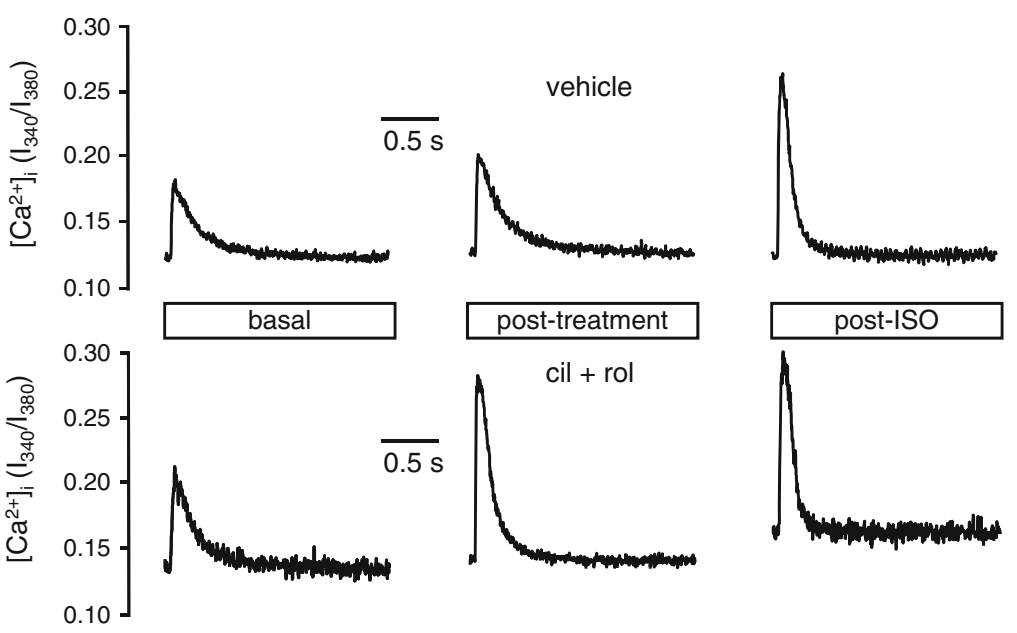

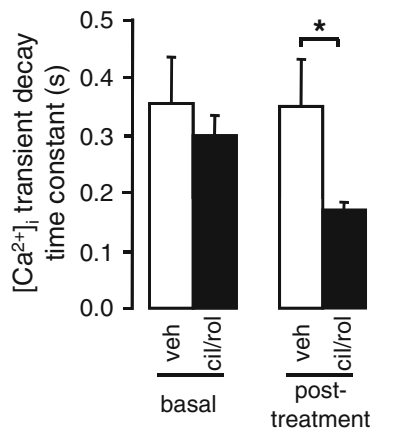

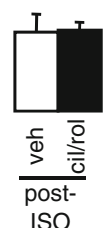


Fig. 6 Inhibition of ET1induced PKD activation requires inhibition of both PDE3 and PDE4. Cultured ARVM were exposed to vehicle, various combinations of EHNA $(10 \mu \mathrm{M})$, cilostamide $(10 \mu \mathrm{M})$ and rolipram $(10 \mu \mathrm{M})$, or ISO $(100 \mathrm{nM})$ for $10 \mathrm{~min}$, followed by vehicle (con) or ET1 (100 nM) for $10 \mathrm{~min}$. a Representative western blots of phosphoSer916 PKD, phosphoSer744/748 PKD, phosphoSer498 HDAC5 and total PKD, as indicated.

b Quantitative data for the ET1induced change in phosphoSer916 PKD, phosphoSer744/748 PKD, and the lower bands from the pSer498 HDAC5 blots $(n=3)$. $* P<0.05$ versus vehicle control
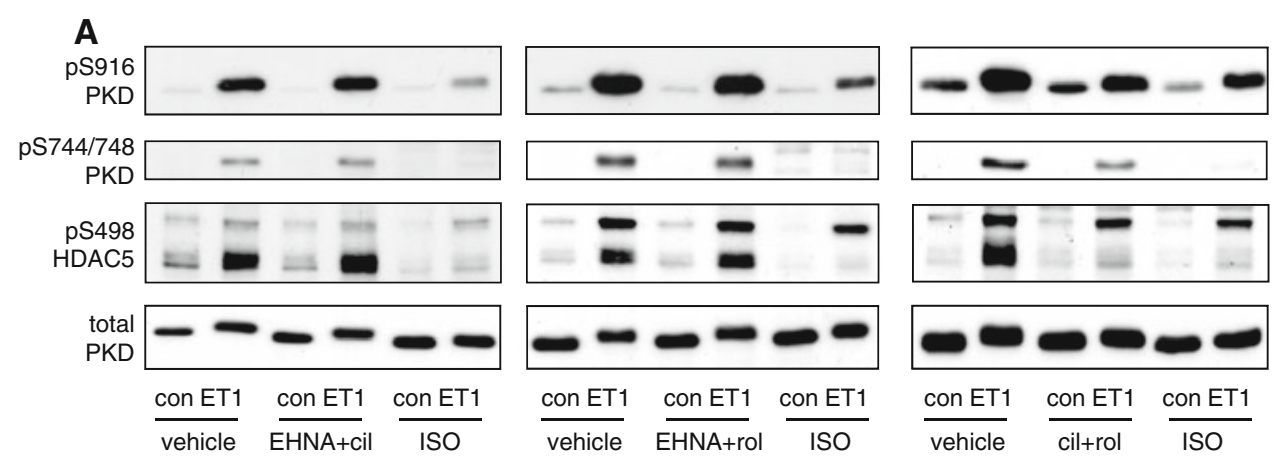

B

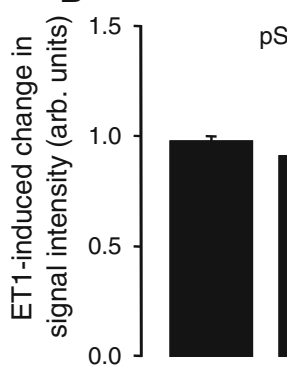

pS916 PKD
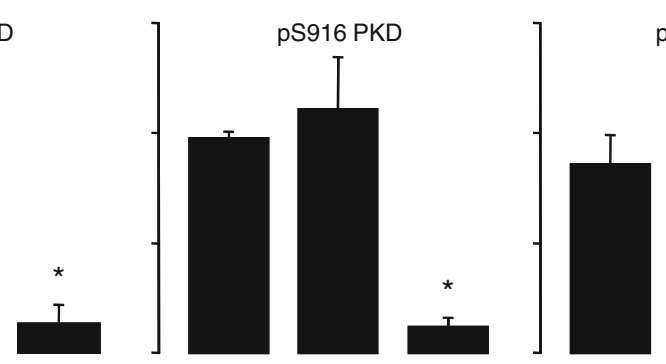

pS916 PKD
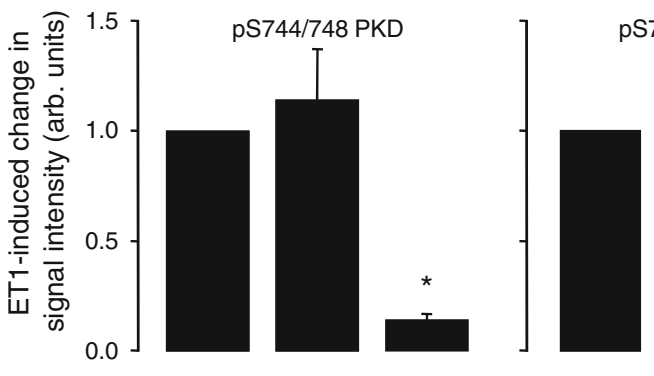

S744/748 PKD
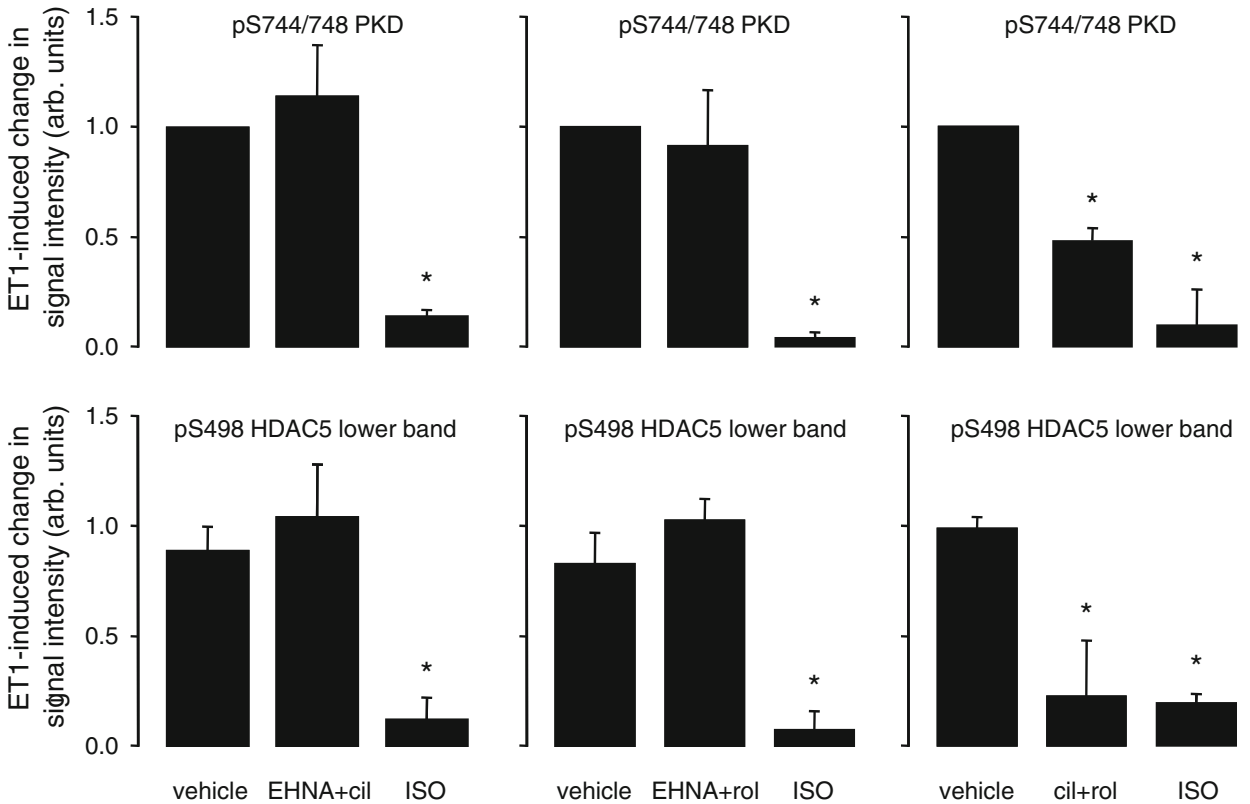

\section{Discussion}

The major findings of this study are that in adult cardiac ventricular myocytes: (1) PDE3 and PDE4 regulate the PKA activity that inhibits ET1-induced PKD activation; (2) the PKA activities that are responsible for PLB phosphorylation at the sarcoplasmic reticulum and cTnI phosphorylation within the sarcomere are also regulated by the same PDE isoforms, PDE3 and PDE4; (3) PDE3 and PDE4 appear to operate in a redundant manner in the above process, such that their individual inhibition has little impact but their combined inhibition markedly increases
PKA activity; (4) PKA activation, either by non-selective PDE inhibition or by $\beta$-AR stimulation, inhibits PKD activation not only by ET1 but also by $\alpha_{1}$-AR stimulation, suggesting that the pertinent inhibitory crosstalk mechanism regulates PKD activation by multiple $\mathrm{G}_{\mathrm{q}} \mathrm{PCR}$ signalling pathways.

The marked inhibitory effect of the non-selective PDE inhibitor IBMX on ET1-induced PKD activation is consistent with our earlier study that first reported a PKAmediated inhibitory crosstalk mechanism in PKD regulation [17], and additionally suggests that PDE inhibition produces a sufficient increase in the PKA activity that 
A
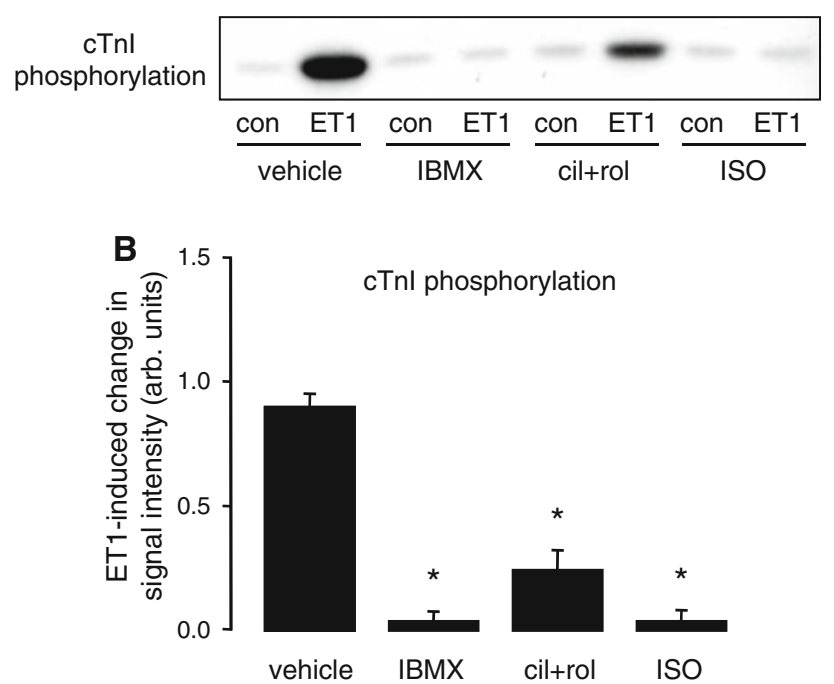

Fig. 7 PDE inhibition regulates ET1-induced PKD activity. Cultured ARVM were exposed to vehicle, IBMX $(100 \mu \mathrm{M})$, cilostamide $(10 \mu \mathrm{M})$ and rolipram $(10 \mu \mathrm{M})$, or ISO $(100 \mathrm{nM})$ for $10 \mathrm{~min}$, followed by vehicle (con) or ET1 $(100 \mathrm{nM})$ for $10 \mathrm{~min}$. PKD was immunoprecipitated from cell lysates and PKD activity was assessed by in vitro kinase assays using cTnI as substrate. a Representative autoradiogram of phosphorylated cTnI. b Quantitative data for the ET1-induced change in cTnI phosphorylation $(n=3) . * P<0.05$ versus vehicle control

inhibits PKD activation, even in the absence of receptormediated adenylate cyclase stimulation. The data we have obtained with isoform-selective PDE inhibitors indicate that PDE3 and PDE4 are the principal isoforms that regulate the pertinent PKA activity, and that these PDE isoforms operate in a redundant manner, such that inhibition of both is required to achieve marked inhibition of PKD activation. Upon exposure of myocytes to ET1, PKCmediated PKD activation is likely to occur at the sarcolemma, following the recruitment of novel PKC isoforms and PKD itself to this compartment through the interaction of their cysteine-rich zinc finger domains with DAG within the lipid bilayer and DAG-mediated PKC activation [2]. On the basis of our current findings, it is reasonable to speculate that PDE3 and PDE4 are responsible for regulating cAMP levels and therefore PKA activity at a sarcolemmal compartment where PKD activation occurs. Consistent with such a possibility, in unstimulated ARVM, the combined inhibition of PDE3 and PDE4 has been shown to increase the activity of a sarcolemmal PKA target, the L-type $\mathrm{Ca}^{2+}$ channel [38], while the individual inhibition of PDE2, PDE3 or PDE4 was without effect [31, 38].

On the other hand, our findings regarding the phosphorylation of known PKA targets in other myocyte compartments argue against specific roles for PDE3 and PDE4 in regulating PKA activity exclusively at a
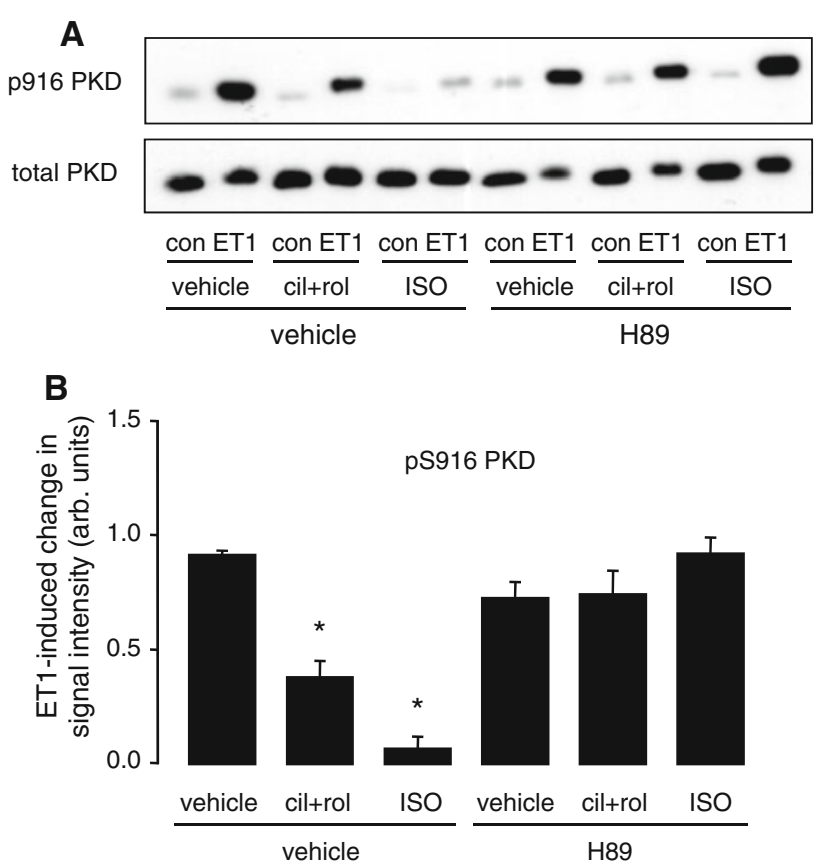

Fig. 8 Combined inhibition of PDE3 and PDE4 inhibits ET1-induced PKD activation through PKA. Cultured ARVM were exposed to vehicle or $\mathrm{H} 89(10 \mu \mathrm{M})$ for $30 \mathrm{~min}$, then vehicle, cilostamide $(10 \mu \mathrm{M})$ and rolipram $(10 \mu \mathrm{M})$, or ISO $(100 \mathrm{nM})$ for $10 \mathrm{~min}$, followed by vehicle (con) or ET1 (100 nM) for 10 min. a Representative western blots of phosphoSer916 PKD and total PKD, as indicated. b Quantitative data for the ET1-induced change in phosphoSer916 PKD $(n=3)$. $* P<0.05$ versus vehicle control

sarcolemmal compartment. We have found that the combined inhibition of PDE3 and PDE4 was sufficient and necessary also for inducing marked phosphorylation of cTnI and PLB at their PKA-targeted residues and producing significant functional effects on myocyte contraction, relaxation and $\mathrm{Ca}^{2+}$ transients, indicating that PDE3 and PDE4 regulate PKA activities additionally at sarcomeric and sarcoplasmic reticular compartments. In this context, it is worth noting that PDE3 and PDE4 are responsible for the vast majority of the total PDE activity in rat cardiac myocytes [26, 31]. It is possible, therefore, that the similar effects of the various PDE inhibitors on PKD activation and $\mathrm{cTnI}$ and PLB phosphorylation in our studies reflect their quantitative effects on total cellular PDE activity, rather than indicating specific roles for PDE3 and PDE4 in regulating PKA activity in defined subcellular compartments. Nevertheless, compartment-specific roles of PDE isoforms may become more evident in the presence of receptor-mediated stimulation of cAMP generation by adenylate cyclase; indeed, while combined inhibition of PDE3 and PDE4 was required to increase L-type $\mathrm{Ca}^{2+}$ channel activity in unstimulated ARVM [38], PDE4 inhibition alone was sufficient to potentiate the induction of such channel activity by sub-maximal $\beta$-AR stimulation 

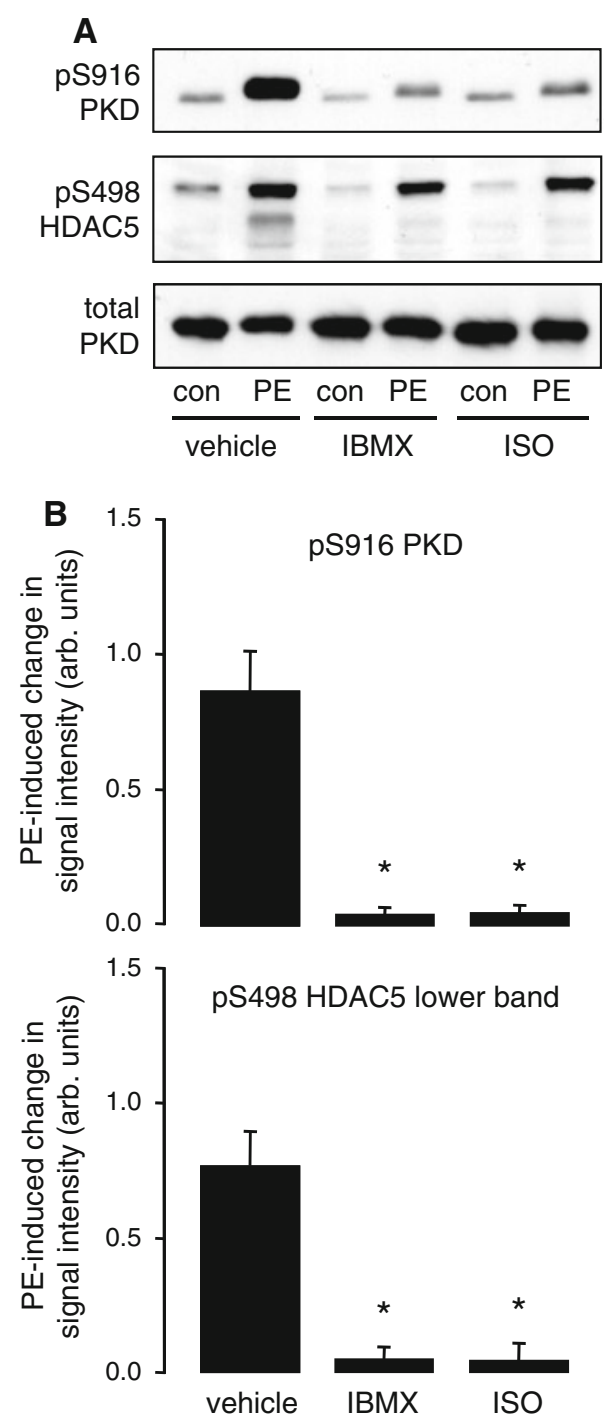

Fig. 9 PE-induced PKD activation is inhibited by pretreatment with IBMX or isoprenaline in ARVM. Cultured ARVM were exposed to vehicle, IBMX $(100 \mu \mathrm{M})$ or ISO $(100 \mathrm{nM})$ for $10 \mathrm{~min}$, followed by vehicle (con) or PE $(10 \mu \mathrm{M})$ for $10 \mathrm{~min}$. a Representative western blots of phosphoSer916 PKD, phosphoSer498 HDAC5 and total PKD, as indicated. b Quantitative data for the PE-induced change in phosphoSer916 PKD, and the lower band from the pSer498 HDAC5 blots $(n=4)$. $* P<0.05$ versus vehicle control

$[23,31,38]$. An interesting corollary finding in our study was that combined PDE3 and PDE4 inhibition and $\beta$-AR stimulation each produced comparable effects on myocyte protein phosphorylation (Fig. 4) and function (Fig. 5), suggesting the presence of considerable basal adenylate cyclase activity in this preparation.

In the course of the present study, we have observed a differential impact of PKA activation, either by PDE inhibition or by $\beta$-AR stimulation, on the appearance of two phosphoprotein moieties that are detected by the phospho-Ser498 HDAC5 antibody. ET1 or PE stimulation markedly increased the phosphorylation of both $140-\mathrm{kDa}$ moiety and 105-kDa moiety, but the phosphorylation status of the 105-kDa protein tracked more faithfully the changes in PKD phosphorylation that arose from PKA activation, consistent with this protein being a downstream target of activated PKD. Although the identities of the two phosphoproteins cannot be ascertained from the present study, it is notable that the motif around the PKD target site in HDAC5, Ser498 (PLSRTQSSPL; underlining indicates the phosphorylated residue) [19], is quite well conserved among other members of the class II HDAC family (e.g. Ser630 in HDAC4, PLSRAQSSPA; Ser457 in HDAC7, PLSRTQSSPA; Ser449 in HDAC9, PLNRTQSAPL). Since the phospho-Ser498 HDAC5 antibody was raised against a synthetic phosphopeptide derived from the HDAC5 sequence around Ser498, such homology suggests that the 140- and 105-kDa phosphoproteins detected by this antibody are likely to be different HDAC isoforms. It would be important to determine how the phosphorylation and nuclear export of specific HDAC isoforms are modulated by the PKA-mediated inhibition of PKD activation that we have delineated in our previous [17] and current work, and to investigate the impact of such inhibitory crosstalk on HDAC-regulated transcriptional reprogramming processes that facilitate cardiac hypertrophy and remodelling upon neurohormonal stimulation [3]. In this context, there is evidence in both adult [32] and neonatal [29] cardiac myocytes that the pro-hypertrophic consequences of $\alpha_{1}$-AR stimulation are attenuated by $\beta$-AR stimulation through a PKA-mediated pathway, although the molecular mechanism(s) underlying this phenomenon have not been deciphered. Furthermore, recent evidence suggests that in failing myocardium, in which the downregulation of $\beta$-AR-mediated signalling pathways is a common phenomenon [25], ET1-induced nuclear export of HDAC5 is potentiated, at least in part through a PKDmediated pathway [6]. These findings support the possibility that PKA-mediated inhibition of PKD activation is of physiological significance, particularly in the context of cardiac hypertrophy and failure.

The upstream molecular mechanism(s) that are responsible for PKA-mediated inhibition of PKD activation in cardiac myocytes remain unknown. Our observation that PKA activation, either by PDE inhibition or by $\beta$-AR stimulation, inhibits PKD activation both by ET1 and by $\mathrm{PE}$ indicates that the inhibitory crosstalk mechanism regulates PKD activation by multiple $\mathrm{G}_{\mathrm{q}} \mathrm{PCR}$ signalling pathways, and suggests that this mechanism must target one or more components that are common to such pathways. Putative PKA targets within the pertinent inhibitory crosstalk mechanism include phospholipase C (PLC) isoforms, since in non-cardiac cells PLC- $\beta_{2}$ and $-\beta_{3}$ have been shown to be directly phosphorylated by PKA, resulting in their inactivation [1, 24]. Furthermore, regulator of $G$ 
protein signalling (RGS) 4 and G protein-coupled receptor kinase 2 (which contains an RGS domain) have also been shown to be directly phosphorylated by PKA, with such phosphorylation increasing their affinity for $\mathrm{G} \alpha_{\mathrm{q}}$ and thereby inhibiting $\mathrm{G}_{\mathrm{q}}$ PCR-mediated stimulation of PLC activity [18]. If such proximal inhibitory mechanisms were operative in cardiac myocytes, however, they would be expected to attenuate not only PKD activation but also additional $\mathrm{G}_{\mathrm{q}}$ PCR-mediated signalling events.

The present study has provided novel information regarding the mechanisms that regulate PKA-mediated inhibition of PKD activation in cardiac myocytes, particularly by revealing the roles of PDE3 and PDE4 and by showing that the inhibitory crosstalk process affects PKD activation by multiple $\mathrm{G}_{\mathrm{q}}$ PCR-mediated pathways. Further work is required to determine both the downstream functional consequences and the upstream molecular mechanisms of such crosstalk.

Acknowledgments This work was supported by a Project Grant from the British Heart Foundation (PG/08/064/25398). We thank Shiney Reji for assistance with myocyte isolation and Ajay Doal for help with preliminary experiments.

\section{Conflict of interest None.}

Open Access This article is distributed under the terms of the Creative Commons Attribution Noncommercial License which permits any noncommercial use, distribution, and reproduction in any medium, provided the original author(s) and source are credited.

\section{References}

1. Ali H, Fisher I, Haribabu B, Richardson RM, Snyderman R (1997) Role of phospholipase $C \beta 3$ phosphorylation in the desensitization of cellular responses to platelet-activating factor. J Biol Chem 272:11706-11709

2. Avkiran M, Rowland AJ, Cuello F, Haworth RS (2008) Protein kinase $\mathrm{D}$ in the cardiovascular system: emerging roles in health and disease. Circ Res 102:157-163

3. Backs J, Olson EN (2006) Control of cardiac growth by histone acetylation/deacetylation. Circ Res 98:15-24

4. Baillie GS (2009) Compartmentalized signalling: spatial regulation of cAMP by the action of compartmentalized phosphodiesterases. FEBS J 276:1790-1799

5. Bardswell SC, Cuello F, Rowland AJ, Gautel M, Walker JW, Kentish JC, Avkiran M (2010) Distinct sarcomeric substrates are responsible for protein kinase D-mediated regulation of cardiac myofilament $\mathrm{Ca}^{2+}$ sensitivity and crossbridge cycling. J Biol Chem 285:5674-5682

6. Bossuyt J, Helmstadter K, Wu X, Clements-Jewry H, Haworth RS, Avkiran M, Martin JL, Pogwizd SM, Bers DM (2008) $\mathrm{CaMKII} \delta$ and PKD overexpression reinforce the HDAC5 redistribution in heart failure. Circ Res 102:695-702

7. Chiu T, Rozengurt E (2001) PKD in intestinal epithelial cells: rapid activation by phorbol esters, LPA, and angiotensin through PKC. Am J Physiol Cell Physiol 280:C929-C942
8. Cuello F, Bardswell SC, Haworth RS, Yin X, Lutz S, Wieland T, Mayr M, Kentish JC, Avkiran M (2007) Protein kinase D selectively targets cardiac troponin I and regulates myofilament $\mathrm{Ca}^{2+}$ sensitivity in ventricular myocytes. Circ Res 100:864-873

9. Cuello F, Snabaitis AK, Cohen MS, Taunton J, Avkiran M (2007) Evidence for direct regulation of myocardial $\mathrm{Na}^{+} / \mathrm{H}^{+}$exchanger isoform 1 phosphorylation and activity by $90-\mathrm{kDa}$ ribosomal $\mathrm{S} 6$ kinase (RSK): effects of the novel and specific RSK inhibitor fmk on responses to alpha1-adrenergic stimulation. Mol Pharmacol 71:799-806

10. de Arcangelis V, Soto D, Xiang Y (2008) Phosphodiesterase 4 and phosphatase 2A differentially regulate cAMP/protein kinase A signaling for cardiac myocyte contraction under stimulation of $\beta_{1}$ adrenergic receptors. Mol Pharmacol 74:1453-1462

11. Di Benedetto G, Zoccarato A, Lissandron V, Terrin A, Li X, Houslay MD, Baillie GS, Zaccolo M (2008) Protein kinase A type I and type II define distinct intracellular signaling compartments. Circ Res 103:836-844

12. Dodge-Kafka KL, Langeberg L, Scott JD (2006) Compartmentation of cyclic nucleotide signaling in the heart: the role of A-kinase anchoring proteins. Circ Res 98:993-1001

13. Fielitz J, Kim MS, Shelton JM, Qi X, Hill JA, Richardson JA, Bassel-Duby R, Olson EN (2008) Requirement of protein kinase D1 for pathological cardiac remodeling. Proc Natl Acad Sci USA 105:3059-3063

14. Harrison BC, Kim M, van Rooij E, Plato CF, Papst PJ, Vega RB, McAnally JA, Richardson JA, Bassel-Duby R, Olson EN, McKinsey TA (2006) Regulation of cardiac stress signaling by protein kinase D1. Mol Cell Biol 26:3875-3888

15. Haworth RS, Cuello F, Herron TJ, Frantzen G, Kentish JC, Gautel M, Avkiran M (2004) Protein kinase D is a novel mediator of cardiac troponin I phosphorylation and regulates myofilament function. Circ Res 95:1091-1099

16. Haworth RS, Goss MW, Rozengurt E, Avkiran M (2000) Expression and activity of protein kinase D/protein kinase $\mathrm{C} \mu$ in myocardium: evidence for $\alpha_{1}$-adrenergic receptor- and protein kinase C-mediated regulation. J Mol Cell Cardiol 32:1013-1023

17. Haworth RS, Roberts NA, Cuello F, Avkiran M (2007) Regulation of protein kinase $\mathrm{D}$ activity in adult myocardium: novel counter-regulatory roles for protein kinase $\mathrm{C} \varepsilon$ and protein kinase A. J Mol Cell Cardiol 43:686-695

18. Huang J, Zhou HZ, Mahavadi S, Sriwai W, Murthy KS (2007) Inhibition of $\mathrm{G} \alpha_{\mathrm{q}}$-dependent PLC- $\beta 1$ activity by PKG and PKA is mediated by phosphorylation of RGS4 and GRK2. Am J Physiol Cell Physiol 292:C200-C208

19. Huynh QK, McKinsey TA (2006) Protein kinase D directly phosphorylates histone deacetylase 5 via a random sequential kinetic mechanism. Arch Biochem Biophys 450:141-148

20. Johannes FJ, Prestle J, Eis S, Oberhagemann P, Pfizenmaier K (1994) $\mathrm{PKC} \mu$ is a novel, atypical member of the protein kinase $\mathrm{C}$ family. J Biol Chem 269:6140-6148

21. Kerfant B-G, Zhao D, Lorenzen-Schmidt I, Wilson LS, Cai S, Chen SRW, Maurice DH, Backx PH (2007) PI3 K $\gamma$ is required for PDE4, not PDE3, activity in subcellular microdomains containing the sarcoplasmic reticular calcium ATPase in cardiomyocytes. Circ Res 101:400-408

22. Leineweber K, Bohm M, Heusch G (2006) Cyclic adenosine monophosphate in acute myocardial infarction with heart failure. Slayer or savior? Circulation 114:365-367

23. Leroy J, Abi-Gerges A, Nikolaev VO, Richter W, Lechene P, Mazet J-L, Conti M, Fischmeister R, Vandecasteele G (2008) Spatiotemporal dynamics of $\beta$-adrenergic cAMP signals and L-type $\mathrm{Ca}^{2+}$ channel regulation in adult rat ventricular myocytes. Circ Res 102:1091-1100 
24. Liu M, Simon MI (1996) Regulation by cAMP-dependent protein kinase of a G-protein-mediated phospholipase C. Nature 382:83-87

25. Lohse MJ, Engelhardt S, Eschenhagen T (2003) What is the role of $\beta$-adrenergic signaling in heart failure? Circ Res 93:896-906

26. Mongillo M, McSorley T, Evellin S, Sood A, Lissandron V, Terrin A, Huston E, Hannawacker A, Lohse MJ, Pozzan T, Houslay MD, Zaccolo M (2004) Fluorescence resonance energy transfer-based analysis of cAMP dynamics in live neonatal rat cardiac myocytes reveals distinct functions of compartmentalized phosphodiesterases. Circ Res 95:67-75

27. Movsesian MA (2002) PDE3 cyclic nucleotide phosphodiesterases and the compartmentation of cyclic nucleotide-mediated signalling in cardiac myocytes. Basic Res Cardiol 97:I83-I90

28. Nikolaev VO, Bunemann M, Schmitteckert E, Lohse MJ, Engelhardt S (2006) Cyclic AMP imaging in adult cardiac myocytes reveals far-reaching $\beta_{1}$-adrenergic but locally confined $\beta_{2}$-adrenergic receptor-mediated signaling. Circ Res 99:10841091

29. Patrizio M, Vago V, Musumeci M, Fecchi K, Sposi NM, Mattei E, Catalano L, Stati T, Marano G (2008) cAMP-mediated $\beta$-adrenergic signaling negatively regulates $\mathrm{G}_{\mathrm{q}}$-coupled receptormediated fetal gene response in cardiomyocytes. J Mol Cell Cardiol 45:761-769

30. Roberts NA, Haworth RS, Avkiran M (2005) Effects of bisindolylmaleimide PKC inhibitors on $\mathrm{p} 90^{\mathrm{RSK}}$ activity in vitro and in adult ventricular myocytes. Br J Pharmacol 145:477-489

31. Rochais F, Abi-Gerges A, Horner K, Lefebvre F, Cooper DMF, Conti M, Fischmeister R, Vandecasteele G (2006) A specific pattern of phosphodiesterases controls the cAMP signals generated by different $\mathrm{G}_{\mathrm{s}}$-coupled receptors in adult rat ventricular myocytes. Circ Res 98:1081-1088

32. Schafer M, Ponicke K, Heinroth-Hoffmann I, Brodde OE, Piper HM, Schluter KD (2001) Beta-adrenoceptor stimulation attenuates the hypertrophic effect of alpha-adrenoceptor stimulation in adult rat ventricular cardiomyocytes. J Am Coll Cardiol $37: 300-307$
33. Snabaitis AK, Muntendorf A, Wieland T, Avkiran M (2005) Regulation of the extracellular signal-regulated kinase pathway in adult myocardium: differential roles of $\mathrm{G}_{\mathrm{q} / 11} . \mathrm{G}_{\mathrm{i}}$ and $\mathrm{G}_{12 / 13}$ proteins in signalling by $\alpha_{1}$-adrenergic, endothelin- 1 and thrombinsensitive protease-activated receptors. Cell Signal 17:655-664

34. Valverde AM, Sinnett-Smith J, van Lint J, Rozengurt E (1994) Molecular cloning and characterisation of protein kinase D: a target for diacylglycerol and phorbol esters with a distinctive catalytic domain. Proc Natl Acad Sci USA 91:8572-8576

35. van Lint J, Sinnett-Smith J, Rozengurt E (1995) Expression and characterisation of PKD, a phorbol ester and diacylglycerol-stimulated serine protein kinase. J Biol Chem 270:1455-1461

36. Vandecasteele G, Rochais F, Abi-Gerges A, Fischmeister R (2006) Functional localization of cAMP signalling in cardiac myocytes. Biochem Soc Trans 34:484-488

37. Vega RB, Harrison BC, Meadows E, Roberts CR, Papst PJ, Olson EN, McKinsey TA (2004) Protein kinases C and D mediate agonist-dependent cardiac hypertrophy through nuclear export of histone deacetylase 5. Mol Cell Biol 24:8374-8385

38. Verde I, Vandecasteele G, Lezoualc'h F, Fischmeister R (1999) Characterization of the cyclic nucleotide phosphodiesterase subtypes involved in the regulation of the L-type $\mathrm{Ca}^{2+}$ current in rat ventricular myocytes. Br J Pharmacol 127:65-74

39. Yuan J, Bae D, Cantrell D, Nel AE, Rozengurt E (2002) Protein kinase $\mathrm{D}$ is a downstream target of protein kinase $\mathrm{C} \theta$. Biochem Biophys Res Commun 291:444-452

40. Zaccolo M (2006) Phosphodiesterases and compartmentalized cAMP signalling in the heart. Eur J Cell Biol 85:693-697

41. Zaccolo M, Pozzan T (2002) Discrete microdomains with high concentration of cAMP in stimulated rat neonatal cardiac myocytes. Science 295:1711-1715

42. Zugaza JL, Sinnett-Smith J, Van Lint J, Rozengurt E (1996) Protein kinase D (PKD) activation in intact cells through a protein kinase $\mathrm{C}$-dependent signal transduction pathway. EMBO J 15:6220-6230 